\title{
Rapid diagnosis of aneuploidy in chromosomes 13, 18, 21, $X$ and $Y$ by quantitative fluorescence-PCR combined with short tandem repeat and fluorescence-labeled homologous gene quantitative-PCR using 4-color fluorescently labeled universal primers
}

\author{
JU LONG, XUEHE YE, XUNJIN WENG, KEPENG FU, LEI SUN and WANRONG PANG \\ Laboratory of Medical Genetics, Maternal and Child Health Care Hospital of Qinzhou, Guangxi 535099, P.R. China
}

Received April 25, 2013; Accepted September 6, 2013

DOI: $10.3892 / \mathrm{mmr} .2013 .1678$

\begin{abstract}
The present study aimed to develop a rapid diagnostic test of aneuploidy in chromosomes 13, 18, 21, $\mathrm{X}$ and $\mathrm{Y}$ through a program combining short tandem repeat (STR) typing with fluorescence-labeled homologous gene quantitative-polymerase chain reaction (fHGQ-PCR), which avoids misjudgment risks by using one method alone. Furthermore, fluorescently labeled universal primers not only ensure the accuracy of the results but also reduces the cost of fluorescent labels. The verification of DNA extracted from samples confirmed by karyotype analysis with quantitative fluorescence (QF)-PCR shows that the results obtained using the QF-PCR program are consistent with the results of karyotype analysis in rapidly diagnosing the aneuploidy of chromosomes 13,18 , 21, $\mathrm{X}$ and $\mathrm{Y}$.
\end{abstract}

\section{Introduction}

Chromosomal abnormalities are hereditary diseases. Approximately $95 \%$ of patients with chromosomal abnormalities suffer the number variation of chromosomes 13,18 , $21, \mathrm{X}$ and $\mathrm{Y}$ (1). The probability of trisomy 21 syndrome is $\sim 1 / 800$ and the predominant symptoms for patients include abnormal appearance and development, and a low IQ; the probability of trisomy 18 is between $1 / 3,000$ and $1 / 8,000$, and the survival rate is $<1$ year for the majority of patients; the probability of trisomy 13 is $\sim 1 / 2$ that of trisomy 18 and the average life expectancy of patients is 3 months (2-4). A number of $\mathrm{X}$ and $\mathrm{Y}$ chromosome abnormalities result in developmental and reproductive disorders. The case numbers of sex chromosome abnormalities diagnosed in the Laboratory of

Correspondence to: $\mathrm{Mr}$. Ju Long, Laboratory of Medical Genetics, Maternal and Child Health Care Hospital of Qinzhou, 1 Anzhou Street, Qinzhou, Guangxi 535099, P.R. China

E-mail: lab@longju.net

Key words: aneuploidy, quantitative fluorescence-polymerase chain reaction, short tandem repeat, fluorescence-labeled homologous gene quantitative-polymerase chain reaction
Medical Genetics (Maternal and Child Health Care Hospital of Qinzhou, Qinzhou, China) are between those of trisomy 21 and those of trisomy 18. Karyotype analysis, a common diagnostic test for chromosomal abnormalities, typically takes 2-4 weeks. Due to the complex operation of the cell culture and karyotype analysis, the risk of failure of the amniotic fluid culture and time taken to obtain results, karyotype analysis is limited in prenatal diagnosis.

Short tandem repeats (STRs) are genetic markers with genetic polymorphism. They are composed of a number of variations in the core sequence repeated units and widely exist in the human genome. The majority of STRs with high polymorphism lie in non-coding regions, free from selection pressure and have adequate allelic products in gene amplification, thus, the markers have been widely used in the fields of forensic medicine, human individual identification and paternity testing $(5,6)$. In the quantitative fluorescence-polymerase chain reaction (QF-PCR) test based on STR typing, the value of STR heterozygous loci of normal individuals in the typing peak is closer to 1:1 while that of STR loci in aneuploid chromosome trisomy in the peak is $2: 1$ or 1:1:1 (1-7). Thus, QF-PCR with the combination of STR analysis may be used to detect chromosome copy numbers. QF-PCR to analyze chromosome karyotype lasts $\sim 24 \mathrm{~h}$. However, misjudgment risks may occur in diagnosing chromosome monomers if the use of STR typing is the only program used to determine aneuploidy diagnosis.

Homologous gene quantitative-PCR (HGQ-PCR) is a method of detecting the ratio between the amplification sequence and the gene copy number. It amplifies homologous nucleic acid fragments with different lengths by using the same primers in different chromosomes (8). For the human chromosome genome, it is possible to diagnose the chromosomal abnormality through its ratios in HGQ-PCR experiments, if the gene fragment chosen only has one homologous sequence of the copy number in a specific chromosome. If the homologous genes are between the autosomes, the quantity of PCR product in the ratio of $1: 1$ is the normal copy number, that in the ratio of $3: 2$ is the trisomy and that in the ratio of $1: 2$ is the monomer. According to the above methods, the HGQ-PCR experiment ratios of homologous sequences between the autosomes and the sex chromosomes are analogized. Thus, the fluorescence-labeled 
HGQ-PCR (fHGQ-PCR) has advantages in chromosome monomer diagnosis.

TERF1 gene encodes the telomeric repeat-binding factor 1 protein (9). Following comparison by basic local alignment search tool (BLAST) of the National Center for Biotechnology Information (NCBI), it was observed that among homologous sequences of TERF1, a segment of homologous sequences is located in chromosomes 13,18 and 21. The segment may be used to detect the copy numbers of chromosomes 13,18 and 21 in fHGQ-PCR, thus it was termed 21-13-18-co1. In addition, it was identified through the BLAST function provided by the NCBI that a segment of homologous sequences are observed in chromosome 18 and $\mathrm{X}$. It is possible to detect the copy numbers of chromosomes 18 and $\mathrm{X}$ by the amplification of the sequence, which we termed X-18-co1. In the present study, 21-13-18-co1, $\mathrm{X}-18$-col and the gender loci Amelogenin (AMXY) which has been widely used in forensic medicine were introduced. The situations of aneuploidy in chromosomes 13, 18, 21 and $\mathrm{X}$ were determined by mutual authentication with STR markers.

In the QF-PCR analysis, the direct labeling method is usually used to label primers. In order to reduce the costs of experimental labeling, 4 universal primers whose fluorescein is labeled at the $5^{\prime}$ end respectively with 6-FAM, VIC, NED and PET were designed. In this study, one end of the amplification primer used the tailing method and tailed sequences were matched with these 4 universal primers respectively. In the PCR amplification test, the amplification primer and labeling primers were added into the reaction tube together to achieve labeling of the amplification product with fluorescence.

The purpose of this study was to develop a type of QF-PCR reagent based on 4-color fluorescently labeled universal primers combined with the fHGQ-PCR program. The reagent was able to diagnose the aneuploidy in chromosomes 13,18 , $21, \mathrm{X}$ and Y accurately and rapidly, and met the requirements of screening chromosome aneuploidies on a large scale.

\section{Materials and methods}

Materials. All experimental samples were obtained from DNA samples stored in the Laboratory of Medical Genetics (Maternal and Child Health Care Hospital of Qinzhou). Samples from 102 normal people were collected by routine tests in the laboratory at random for the pre-experimental study, to validate the method of fHGO-PCR. Subsequent to the preliminary experiment, samples confirmed by karyotype analysis were used for PCR amplification tests following the extraction of DNA. In this study, 3 trisomy 21 samples, 2 trisomy 18 samples, 1 trisomy 13 sample, 3 aneuploidy in chromosome $\mathrm{X}$ or $\mathrm{Y}$ samples and 10 normal samples were used to verify the QF-PCR system. All aneuploidy samples were extracted from cultured amniotic fluid cells, and all normal DNA samples were extracted from uncultured blood cells. Lab-Aid DNA mini extraction kit (Bio-V, Xiamen, Fujian, China) was used for DNA extraction. The study was approved by the ethics committee of Maternal and Child Health Care Hospital of Qinzhou and written informed consent was obtained from the patients.

Design and verification of multiple-tailed primers. An experiment was designed to verify the feasibility of the QF-PCR based on the following universal primers: AMXY-6-FAM-F (5'-6-FAM-CCC TGG GCT CT G TAA AGA ATA GTG-3') and CoA1-6-FAM (5'-6-FAM-CT C GAC ACG CAT CTG CTC AG-3'), whose fluorescein are labeled at the 5' end by directly using 6-FAM, AMXY-R (5'-ATC AGA GCT TAA ACT GGG AAG CTG-3') unlabeled and CoA1-AMXY-F (5'-CTC GAC ACG CAT CTG CTC AG-CCC TGG GCT CTG TAA AGA ATA GTG-3') tailed as multiple-tailed primers. Four reaction tubes were used in the method. The $\mathrm{V} 1$ reaction tube contained AMXY-6-FAM-F and AMXY-R primers, and V2-V4 reaction tubes contained CoA1-AMXY-F, AMXY-R and CoA1-6-FAM primers. The PCR reagent used was 2X GoldStar Taq Mastermix (CoWin Biosciences, Inc., Beijing, China) and the volume of the reaction liquid was $25 \mu \mathrm{l}$. The reaction liquid contained $0.5 \mu \mathrm{l}$ of every primer $(10 \mu \mathrm{M})$ and $1 \mu \mathrm{l}$ DNA with the concentration of $50 \mathrm{ng} / \mu \mathrm{l}$ in male samples. PCR amplification was conducted as follows: $95^{\circ} \mathrm{C}$ for $11 \mathrm{~min}$, then a variable number of cycles (V1 and V2, 28 cycles; V3, 30 cycles; V4, 32 cycles) of $95^{\circ} \mathrm{C}$ for $30 \mathrm{sec}$, $59^{\circ} \mathrm{C}$ for $30 \mathrm{sec}$ and $72^{\circ} \mathrm{C}$ for $30 \mathrm{sec}$, with a final extension at $72^{\circ} \mathrm{C}$ for $20 \mathrm{~min}$. ABI 9700 (Applied Biosystems, Foster City, CA, USA) was used to conduct PCR. Product analysis was performed by ABI 3130 (Applied Biosystems), LIZ 500 (Applied Biosystems) was used as a size standard and degeneration was conducted using deionized formamide (HiDi). In 3 analysis wells in a 96 -well plate, V1 products $(0.3 \mu \mathrm{l})$ were added to each well and combined with $0.3 \mu \mathrm{l}$ V2-V4 products separately and the analysis procedure began following mixing. A comparison of the quantity of product of V1 and that of V2-V4 tubes was conducted. Subsequent to this, the shape of the product of V1 was compared with that of the product of V2-V4. Following verification, another 3 universal primers termed CoA2 (5'-VIC-CTC GAC ACG CAT CTG ACG TT-3'), CoA3 (5'-NED-CTC GAC ACG CAT CTG GCG AA-3') and CoA4 (5'-PET-CTC GAC ACG CAT CTG CTA $C$ - $-3^{\prime}$ ) were designed. The primers were labeled at the $5^{\prime}$ end using VIC, NED and PET. CoA2, CoA3 and CoA4 were synthesized by Life Technologies (Carlsbad, CA, USA), and the rest of the primers were synthesized by Sangon (Shanghai, China).

Selection of STR loci. Based on the literature (10-14), 16 STR loci and the gender loci AMXY were selected to complete the construction of the experimental system. STR loci with universal primer CoA1, included D21S11, D18S1002, D21S2055 and D21S1412; STR loci with universal primer CoA2 included D18S391, D18S51 and D18S386; STR loci with universal primer CoA3, included AMXY D13S895 SRY, D13S631, D13S258, D21S1270 and D13S634; and STR loci with universal primer CoA4, included DXS9902, X22 and XHPRT.

Design of $f H G Q-P C R$ primers. According to the BLAST results, 21-13-18-col and X-18-col multiple-tailed primers, the 21-13-18-co1 multiple-tailed primer with universal primer CoA4 and the X-18-col multiple-tailed primer with universal primer CoA3. The 21-13-18-col amplification primers were as follows: Forward: 5'-CTC GAC ACG CAT CTG CTA CCA GAC AGT AGG CTG CCT CAT G-3' and reverse: 5'-GAA TAT CAT TTC TCA GTC CCA AGC C-3' for 21-13-18-co1; 
A

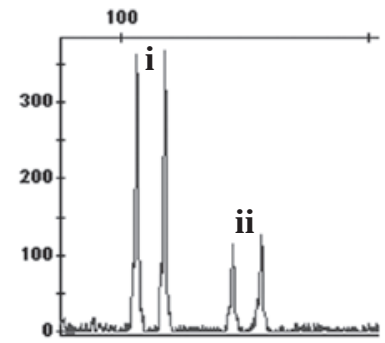

B

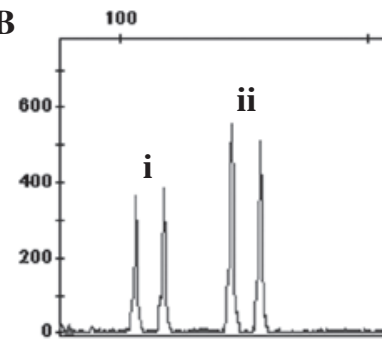

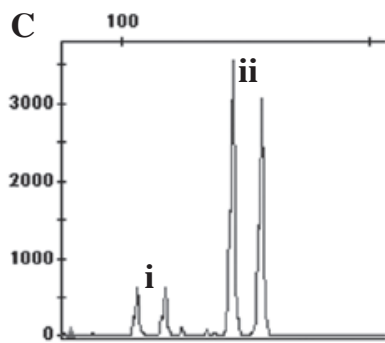

Figure 1. QF-PCR system for (A) 28 cycles; (B) 30 cycles; (C) 32 cycles based on universal primers. Ai, Bi and Ci peaks show the product of V1; Aii peaks show the product of V2; Bii peaks show the product of V3; Cii peaks show the product of V4. The X-axis represents the fragment length and the Y-axis is the peak value. A, B and C show two groups of product peaks separately (two peaks per group). The left peak set was directly fluorescent labeled and the right peak set was fluorescent tail labeled. QF-PCR, quantitative fluorescent-polymerase chain reaction.

and forward: 5'-CTC GAC ACG CAT CTG GCG AAT GGC CAA GGG GTT TAA CTC-3' and reverse: 5'-CTT GTC TTT CTT TAG GCC AAA T-3' for 18-X-col.

Single-tube QF-PCR system and procedures. The single-tube QF-PCR system that was designed included multiple-tailed primers of 16 STR loci, multiple-tailed primers of AMXY, 21-13-18-co1, X-18-col and 4 universal primers used for fluorescent labels. The PCR reagent used was $2 \mathrm{X}$ GoldStar Taq Mastermix and the volume of the PCR reaction liquid was $25 \mu \mathrm{l}$. The individual primer concentrations used in this system were as follows: $0.4 \mu \mathrm{M}$ D18S51, $0.12 \mu \mathrm{M}$ D18S391, $0.32 \mu \mathrm{M}$ D18S386, 0.2 $\mu \mathrm{M}$ D21S2055, 0.4 $\mu \mathrm{M}$ D21S11, $0.16 \mu \mathrm{M}$ D21S1412, $0.12 \mu \mathrm{M}$ AMXY, $0.16 \mu \mathrm{M}$ D18S1002, $0.4 \mu \mathrm{M} X-18-c o 1,0.4 \mu \mathrm{M}$ D13S895, $0.4 \mu \mathrm{M}$ D13S631, $0.4 \mu \mathrm{M}$ D13S258, 0.4 $\mu \mathrm{M}$ D13S634, 0.4 $\mu \mathrm{M}$ D21S1270, 0.12 $\mu \mathrm{M}$ SRY, 0.2 $\mu \mathrm{M}$ 21-13-18-co1, $0.36 \mu \mathrm{M}$ XHPRT, $0.4 \mu \mathrm{M}$ X22 and $0.2 \mu \mathrm{M}$ DXS9902. The concentration of each fluorescently labeled universal primer was $0.4 \mu \mathrm{M}$ and the template DNA was 50-100 ng. An ABI 9700 amplification instrument was used in the PCR experiment. PCR amplification was conducted as follows: $95^{\circ} \mathrm{C}(11 \mathrm{~min})$, then 29 cycles at $95^{\circ} \mathrm{C}$ $(30 \mathrm{sec}) / 59^{\circ} \mathrm{C}(30 \mathrm{sec}) / 72^{\circ} \mathrm{C}(30 \mathrm{sec})$, and a final extension at $72^{\circ} \mathrm{C}$ for $20 \mathrm{~min}$. The products were analyzed using an ABI 3130 genetic analyzer and the size standard was LIZ 500. Our DNA samples were confirmed by karyotype analysis in the laboratory.

\section{Results}

Results of multiple-tailed primers. In the QF-PCR experiments based on multiple-tailed primers for demonstration, the results showed that the product peak value of 30 reaction cycles with multiple-tailed reaction tubes is similar with that of 28 reaction cycles with fluorescence-tailed reaction tubes (Fig. 1). In the present study, the hetero-peaks were not observed in the region outside of the target band. Therefore, the QF-PCR experiment based on universal primers is effective.

Validation of the method of fHGO-PCR. Among amplification products of 21-13-18-col, the amplification product of chromosome 21,13 and 18 were 114,120 and $121 \mathrm{bp}$, respectively. As there is only $1 \mathrm{bp}$ difference between 120 and $121 \mathrm{bp}$, the two peaks in the peak figure tend to merge into one in certain experiments. However, this does not affect the results, as the ratio between $114 \mathrm{bp}$ on the left and 120bp and $121 \mathrm{bp}$ on the right approaches 1:1. When the DNA source is the entity of trisomy 21, the ratio between the left peak value and the right peak value approaches 1.5:1. When the source of DNA is the entity of trisomy 13 or 18 , the ratio between the left peak value and the right peak value is closer to 1:1.5. The common validation with STR loci distinguishes whether the samples exhibit trisomy 13 or 18 .

Among the amplification products of X-18-col, the products for the $\mathrm{X}$ chromosome and chromosome 18 are $496 \mathrm{bp}$ and $511 \mathrm{bp}$, respectively. In this locus amplification of DNA from normal males, $496 \mathrm{bp}$ represents the left products of the $\mathrm{X}$ chromosome, while $511 \mathrm{bp}$ is representative of the right products of chromosome 18 . Thus, their ratios tend to be 1:2. However, in normal female samples, the ratio between the left product peak value and the right product peak value is close to $1: 1$. In male samples of trisomy 18 , the ratio between the left product peak value and the right product peak value nearly approaches 1:3, while in the female samples of trisomy 18 that increases to 2:3. This locus may also be applied with AMXY to reduce the probablility of misjudgment from STR markers diagnosing $(45, \mathrm{X}),(48, \mathrm{XXXX)}$ ) and other sex chromosome abnormalities. If AMXY only analyzes the X-peak and the ratio between the $\mathrm{X}$ chromosome and chromosome 18 in the 18 - $\mathrm{X}$-col is $1: 2$, the individual is $(45, \mathrm{X})$; if AMXY simultaneously analyzes the product peak at the ratio of $1: 1$ and the ratio between the $\mathrm{X}$ chromosome and chromosome 18 in the 18 -X-col is $1: 1$, the individual is $(48, \mathrm{XXYY})$. The diagnosis methods of other sex chromosome anomalies are analogized based on the ratio association.

With the loci of AMXY (Fig. 2A), the ratio between the X peaks and $\mathrm{Y}$ peaks is between 1.03 and 0.92 (male samples, $\mathrm{X}$ peak value as 1). With the loci of X-18-col (Fig. 2B), the ratio between the $\mathrm{X}$ peaks and chromosome 18 peaks is between 2.27 and 1.50 (male samples, $X$ peak value as 1 ) or between 1.31 and 1.04 (female samples, $X$ peak value as 1 ). With the loci of 21-13-18-col (Fig. 2C), the ratio between chromosome 21 peaks and chromosome 13/18 peaks is between 1.60 and 0.97 (chromosome 21 peak value as 1).

Results of the single-tube QF-PCR system. The results (Fig. 3) of the single-tube QF-PCR system that was designed are consistent with the results of the karyotype analysis in the experiment of rapidly diagnosing the aneuploidy in chromosomes $13,18,21, \mathrm{X}$ and $\mathrm{Y}$. 

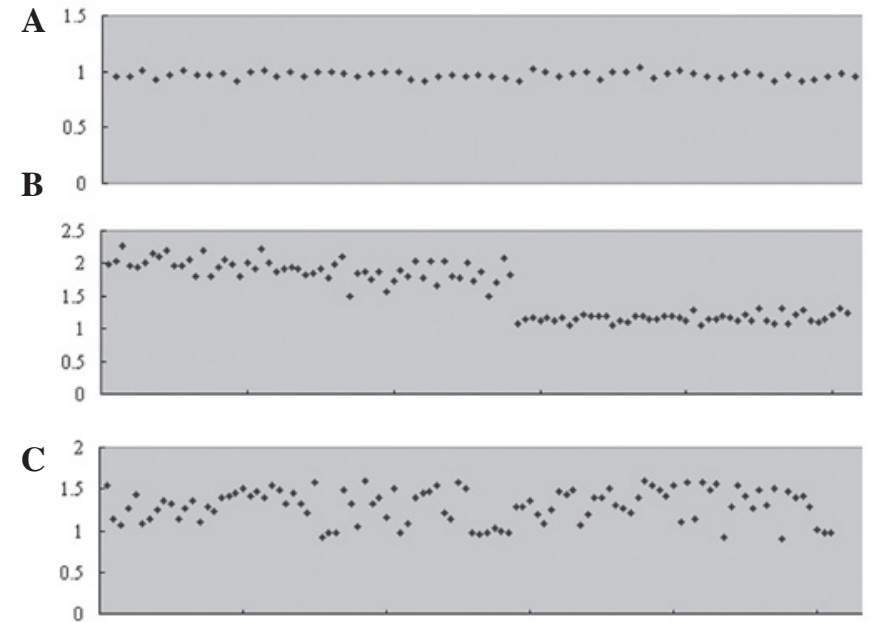

Figure 2. The ratios between allele peaks of fHGO-PCR with 102 normal samples. (A) Loci of AMXY, the ratio between X peaks and Y peaks (male samples, $\mathrm{X}$ peak value as 1); (B) loci of X-18-col, the ratio between $\mathrm{X}$ peaks and chromosome 18 peaks (56 male samples on left side and 46 female samples on right side, $\mathrm{X}$ peak value as 1); (C) loci of 21-13-18-col, the ratio between chromosome 21 peaks and chromosome 13/18 peaks (chromosome 21 peak value as 1). fHGO-PCR, fluorescence-labeled homologous gene quantitative-polymerase chain reaction; AMXY, gender loci Amelogenin.

\section{Discussion}

Studies have shown that the single-tube multiplex QF-PCR is effective in the majority of diagnoses of the aneuploidy in chromosomes $13,18,21, \mathrm{X}$ and $\mathrm{Y}(1,2,10,12)$. The majority of studies used the single-tube multiplex STR typing technique which designs fluorescent labels to diagnose the aneuploidy in chromosomes 13, 18, 21, X and Y through STR loci typing results. However, there exist misjudgment risks in diagnosing the aneuploidy in chromosomes $13,18,21, \mathrm{X}$ and $\mathrm{Y}$, and the monomer in chromosomes $13,18,21, \mathrm{X}$ and $\mathrm{Y}$ using the single-tube multiplex STR typing technique alone. If labels from chromosomes $13,18,21$ or a chromosome relevant to chromosome $\mathrm{X}$ are homozygous, testers will not be able to diagnose whether the chromosome is a monomer by STR typing or these labels are only produced by the same allele. Cirigliano et al (7) suggested Turner's syndrome (45, X) and other monomers may be diagnosed by the quantitative method with the use of different fluorescence-labeled STR loci of similar size fragments; however, there may also be a risk of misjudgment when using this method as the fluorescence intensity varies with different fluoresceins; it is debatable as it used different PCR primers to quantify the amplification products of different loci, as differences in the quantity of PCR product between similar product length STR loci are not uncommon in day-to-day paternity testing cases.

fHGQ-PCR is a method of detecting the copy number of homologous genes. In the present study of fHGQ-PCR, AMXY gene loci were used, which have been widely used in detecting genders of DNA sequences obtained from individuals. Based on the principle of fHGQ-PCR, this method may aid in increasing the reliability of determining the quantity of the $\mathrm{X}$ chromosome by looking for the $\mathrm{X}$ chromosome or the $\mathrm{Y}$ chromosome sequence homologous with autosomes. In the present study 21-13-18-col, X-18-col and AMXY loci

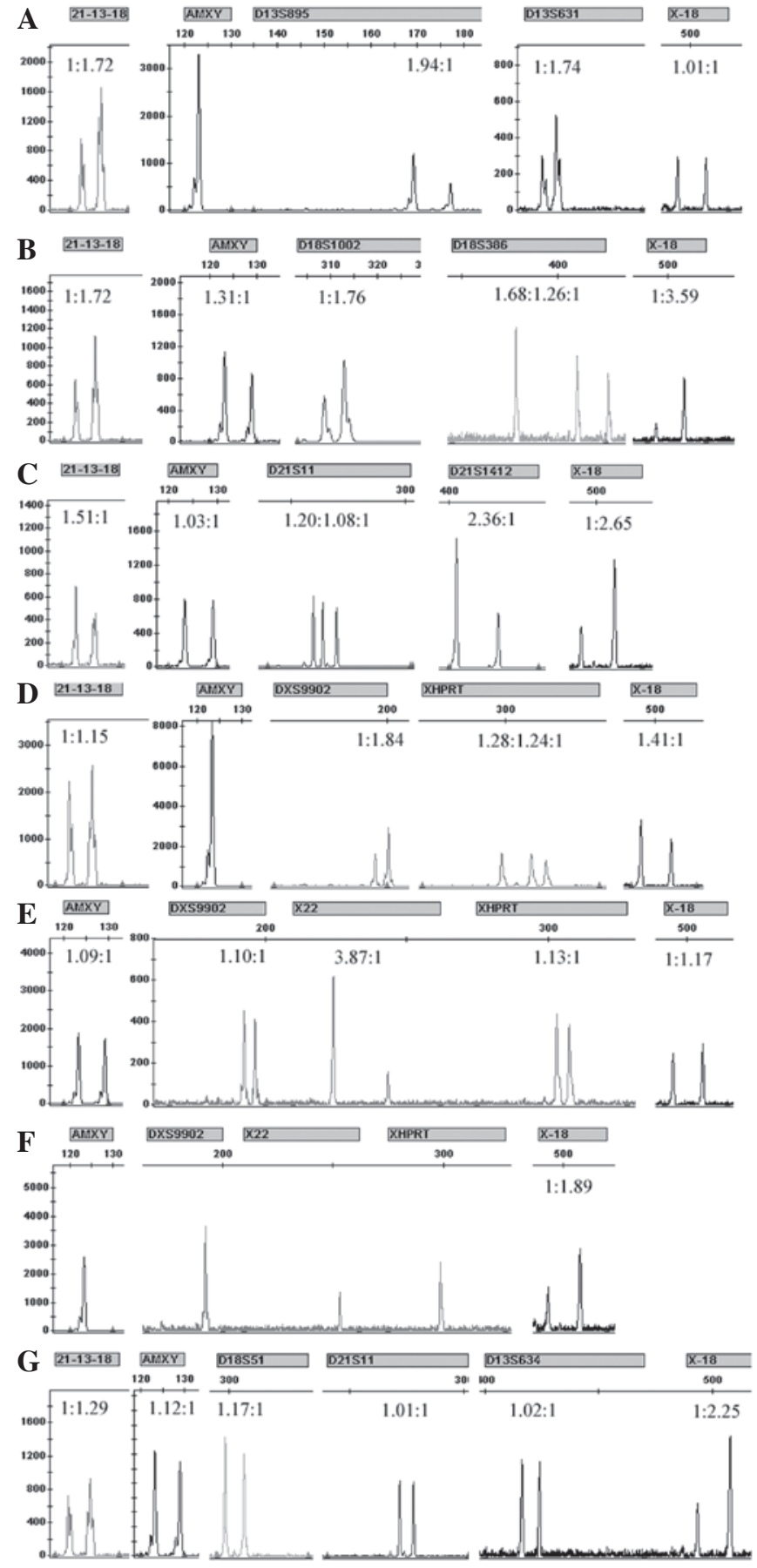

Figure 3. QF-PCR results. The ratio in the peak figure is that between allele peaks. The resulting figure and corresponding karyotypes are as follows: (A) 47,XX+13; (B) 47,XY+18; (C) 47,XY+21; (D) 47,XXX; (E) 48,XXYY; (F) 45X; (G) 46,XY. QF-PCR, quantitative fluorescent-polymerase chain reaction.

were introduced to diagnose aneuploidy in chromosomes 13 , 18, 21, X and Y. According to the results shown in Fig. 2, the ratio between the allele peaks in AMXY or X-18-col vary compared with the perfect theoretical ratio. Although the ratio between the allele peaks in 21-13-18-col are marginally higher than the theoretical ratio for the two peaks for chromosome 13 and 18 , and the peaks merge into one in certain experiments, there remains a reference value in the diagnosis of aneuploidy in chromosomes 13,18 and 21 when the ratio between chromosome 21 peaks and chromosome $13 / 18$ peaks is $>1.60$ or 
$<0.8$ (chromosome 21 peak value as 1), its effect is significant Nevertheless, Shadrach et al (15) and Roffey et al (16) respectively demonstrated the misjudgment cases resulting from the gene loci AMXY mutation and a study by Huang et al (17) also confirmed the significant influence of genetic mutations in PCR amplification efficiency. Moreover, the study of the heterozygous amplification quantity of heterozygous alleles shows the amplification quantity does not produce the theoretical ratio but a ratio range (18). Therefore, in the STR experiment, the quantity of the heterozygous allele in normal individuals was not strictly $1: 1$ and the quantity of the trisomy heterozygous gene was not strictly $1: 1: 1$ or $1: 2$, but varies compared with the perfect theoretical ratio. As the ratio between genetic mutations and the quantity of product does not follow the perfect theoretical ratio, there are misjudgment risks in diagnosing chromosome aneuploidy using fHGQ-PCR alone.

This study concerning the comprehensive application of STR amplification and fHGQ-PCR techniques achieves results through mutual authentication between STR loci and fHGQ-PCR. It neutralizes the disadvantages of simply using STR or fHGQ-PCR to diagnose the aneuploidy in chromosomes $13,18,21, \mathrm{X}$ and $\mathrm{Y}$, and ensures the reliability of the diagnosis results. Compared with the 2-4 weeks required by conventional karyotype analysis, using the QF-PCR program in the present study markedly reduced the time, as the program only takes $6 \mathrm{~h}$ to obtain the results when the DNA samples have been extracted. In addition, the design of four-color fluorescently labeled universal primers not only ensures the accuracy of the experimental results, but also reduces the costs of the QF-PCR system.

\section{References}

1. Ochshorn Y, Bar-Shira A, Jonish A and Yaron Y: Rapid prenatal diagnosis of aneuploidy for chromosomes $21,18,13$, and $\mathrm{X}$ by quantitative fluorescence polymerase chain reaction. Fetal Diagn Ther 21: 326-331, 2006.

2. Andonova S, Vazharova R, Dimitrova V, Mazneikova V, Toncheva D and Kremensky I: Introduction of the QF-PCR analysis for the purposes of prenatal diagnosis in Bulgaria - estimation of applicability of 6 STR markers on chromosomes 21 and 18. Prenat Diagn 24: 202-208, 2004.

3. Palomaki GE, Deciu C, Kloza EM, Lambert-Messerlian GM, Haddow JE, Neveux LM, et al: DNA sequencing of maternal plasma reliably identifies trisomy 18 and trisomy 13 as well as Down syndrome: an international collaborative study. Genet Med 14: 296-305, 2012.
4. Lin HY, Lin SP, Chen YJ, et al: Clinical characteristics and survival of trisomy 18 in a medical center in Taipei, 1988-2004. Am J Med Genet A 140: 945-951, 2006.

5. Shin SH, Yu JS, Park SW, et al: Genetic analysis of 18 X-linked short tandem repeat markers in Korean population. Forensic Sci Int 147: 35-41, 2005.

6. Kim UK, Chae JJ, Lee SH, et al: Molecular diagnosis of Duchenne/Becker muscular dystrophy by polymerase chain reaction and microsatellite analysis. Mol Cells 13: 385-388, 2002.

7. Cirigliano V, Ejarque M, Fuster $\mathrm{C}$ and Adinolfi M: X chromosome dosage by quantitative fluorescent PCR and rapid prenatal diagnosis of sex chromosome aneuploidies. Mol Hum Reprod 8: 1042-1045, 2002.

8. Lee HH, Chang JG, Lin SP, Chao HT, Yang ML and Ng HT: Rapid detection of trisomy 21 by homologous gene quantitative PCR (HGQ-PCR). Hum Genet 99: 364-367, 1997.

9. Shen M, Haggblom C, Vogt M, Hunter T and Lu KP: Characterization and cell cycle regulation of the related human telomeric proteins Pin2 and TRF1 suggest a role in mitosis. Proc Natl Acad Sci USA 94: 13618-13623, 1997.

10. Baig S, Ho SS, Ng BL, Chiu L, Koay ES, Leow GH, et al: Development of quantitative-fluorescence polymerase chain reaction for the rapid prenatal diagnosis of common chromosomal aneuploidies in 1,000 samples in Singapore. Singapore Med J 51: 343-348, 2010.

11. Liang W, Zhang L, Chen G, Xin J, Liao M and Wu MY: Allele distributions for D21S1435 and D21S2055 loci in two Chinese populations. J Forensic Sci 47: 667-668, 2002.

12. Schmidt W, Jenderny J, Hecher K, Hackelöer BJ, Kerber S, Kochhan L and Held KR: Detection of aneuploidy in chromosomes X, Y, 13, 18 and 21 by QF-PCR in 662 selected pregnancies at risk. Mol Hum Reprod 6: 855-860, 2000.

13. Vats KR, Ishwad C, Singla I, Vats A, Ferrell R, Ellis D, et al: A locus for renal malformations including vesico-ureteric reflux on chromosome 13q33-34. J Am Soc Nephrol 17: 1158-1167, 2006.

14. Asmundo A, Perri F and Sapienza D: Allele distribution of two X-chromosomal STR loci in a population from Sicily (Southern Italy). International Congress Series 1288: 346-348, 2006.

15. Shadrach B, Commane M, Hren C and Warshawsky I: A rare mutation in the primer binding region of the amelogenin gene can interfere with gender identification. J Mol Diagn 6: 401-405, 2004.

16. Roffey PE, Eckhoff CI and Kuhl JL: A rare mutation in the amelogenin gene and its potential investigative ramifications. J Forensic Sci 45: 1016-1019, 2000.

17. Huang MM, Arnheim N and Goodman MF: Extension of base mispairs by Taq DNA polymerase: implications for single nucleotide discrimination in PCR. Nucleic Acids Res 20: 4567-4573, 1992

18. Leclair B, Frégeau CJ, Bowen KL and Fourney RM: Systematic analysis of stutter percentages and allele peak height and peak area ratios at heterozygous STR loci for forensic casework and database samples. J Forensic Sci 49: 968-980, 2004. 